\section{Biology of the}

Neonate

Acute hypoxia 195

Alveolar proteinosis 314

Amniotic fluid 145

Analgesia 243

Antioxidant defenses, erythrocytes 188

Apoptosis 90

Arrhythmias, Holter recordings 229

Asphyxia 105

Attention deficit/hyperactivity disorder 203

Betamethasone 121

Birth defects 73

Blood-brain barrier 138

Brain 51, 221

Bronchopulmonary dysplasia 173 , 305

Cardiac troponins 269

CD3 61

CD4 61

CD25 3

CD40L 3

CD45RA 61

Cell membrane 290

Cerebral hypoxemia-ischemia-hypercapnia 105

- palsy 305

Cerebrovascular reactivity 112

Chemical teratogenesis 73

Chronic lung disease 273

Ciprofloxacin, nosocomial infections 263

Cisapride treatment, infants 229

Clotting 82

Coagulase-negative staphylococcus 151

Collectins 326

Cord blood 3

Coronavirus 293

Cortisol 26

C-reactive protein 237

Dexamethasone 94

Digestive enzymes 94

Dipropylenetriamine 195

Down syndrome 167

Endogenous distress 243

Erythropoietin 51, 138, 237

Exocytosis 299

Extremely low birth weight infants 68
Fetal asphyxia, rat 217

- distress 61

- hypoxia, rat 217

- rat model, hypoxia 217

Free radicals 184, 221

Gastrointestinal motility 11

Gender, malnourishment 256

Gene-environment interactions 73

Gestational diabetes 237

Glucocorticoids 82

Glutathione recycling 188

Graded hypoxia 128

Growth hormone 15

Heart development, early-life malnourishment 256

Hematological parameters, smoking 249

Holter recordings, cisapride treatment 229

Homocysteine 249

Hypercapnia 112

Hypoxia 42, 51, 112, 237

Hypoxic-ischemic encephalopathy 15

Immunity 326

Infant, newborn 314

-, premature 305,319

Infection 305

Inflammation 82

Information processing, very-low-birthweight children 203

Insulin-like growth factor 115

Interferon gamma 55

Interleukins 3, 55, 179

Interstitial lung disease 314

Intestinal atrophy 145

- development 145

- morphology 94

- villi 145

Ischemia 51, 221

Ischemia-reperfusion injury 90,151

Lactic acidosis 210

Lamellar bodies 285, 299

Laser-Doppler flowmetry, cerebral hemodynamics 112

Leukomalacia, periventricular 305

Lipid peroxidation 221

Low birth weight 203

Lung injury 290
Malondialdehyde 121

Mechanical stress 290

Membrane fusion 285, 299

Metabolic bone disease, prematurity 225

$\mathrm{N}$-methyl- $D$-aspartate receptor subunits, phosphorylation/dephosphorylation 128

Methylmalonic acid 249

Microcirculation 105

Microdialysis 105

Microvasculature, chronic lung disease 273

Mitochondrial diseases 210

Myocardial metabolism 42

- performance 42

Near-infrared spectroscopy, cerebral hemodynamics 112

Necrotizing enterocolitis 159

Neonates, brain 128

-, cytokine secretion imbalance 55

- distress 243

-, erythrocytes 188

-, gastrointestinal tract anomalies 145

,- heart 42

-, intensive care 243

-, seizures 68

-, smoking mothers 249

Neurodevelopment 68

Neuroprotection 138

Nitric oxide 51, 195

NONOate 195

Nosocomial infection, newborns 263

Nucleated red blood cells, rat hypoxia model 217

Nutrition, gender 256

Observer agreement, gastroesophageal reflux 11

Olive oil, lipid peroxidation 184

Ouabain-like factor, preterm versus mature newborns 155

Oxidant injury 121

Oxygen 105, 121

- insufficiency 42

- saturation 319

Oxygenation index 243

Parenteral nutrition 184

Patent ductus arteriosus 32

Persistent pulmonary hypertension, newborn 195 
Pharmacokinetics, recombinant erythropoietin 138

Plasma amino acids 173

Platelet-activating factor 159

Polymorphism, IL-1 ra gene 179

Preterm infants 37, 121, 155, 173, 179

- -, chronic respiratory insufficiency 273

- -, pain 26

Proinflammatory cytokines 167

Propofol 221

Pulmonary alveoli 299

- function measurements 32

- hypertension 173

- stretch 299

- surfactants 299, 314, 326

Pulse oximetry 319

- rate 26

Pyruvate metabolism 210

Red blood cell redox potential 188

Redox status 210
Respiratory distress syndrome 82 Response time, information processing 203

Sedation 243

Sepsis 179

Severe acute respiratory syndrome 293

Small intestine 90,151

Smoking mothers, newborn infants 249

Sound, tibial speed 225

Soybean oil, lipid peroxidation 184

Spinal fluid 138

Status epilepticus 68

Stroke 221

Sucrose analgesia 26

Surfactant proteins 285,326

Surgical ligation, patent ductus arteriosus 32

Tachycardia 42

Teratogens 73

Tissue damage 184
T lymphocytes, CD45RA expression 61

Transcutaneous bilirubin measurements, accuracy 21

Transient myeloproliferative disorder 167

Twins, umbilical cord blood troponin levels 269

Type 1 diabetes 237

Umbilical artery gas variables 237

Urinary calcium/sodium excretion 37

Validation, multiple intraluminal impedance 11

Ventilation 82

Very-low-birth-weight newborns 210

Vitamin status, smoking 249

White matter damage 305

Xylose absorption 94 